\title{
Dermatology
}

Dermatology 2008;217:378-379

DOI: $\underline{10.1159 / 000158638}$

\section{Prurigo in Dermatomyositis}

\section{Tetsuya Higuchi, Takahiro Satoh, Hiroo Yokozeki}

Department of Dermatology, Tokyo Medical and Dental University, Tokyo, Japan

Key Words

Dermatomyositis $\cdot$ Prurigo

\section{Introduction}

Dermatomyositis is a disease that has characteristic skin eruptions with or without proximal myopathy. Common cutaneous manifestations include Gottron's sign, Gottron's papules, heliotrope rash, poikiloderma, flagellate erythema and mechanic's hands $[1,2]$. Less common cutaneous manifestations, such as vesiculobullous lesions [3], panniculitis [4], plaque-like mucinosis [5], a pityriasis-rubra-pilaris-like eruption $[6,7]$ and seborrheic area erythema [8], are also reported.

We report 2 cases of prurigo-like eruptions associated with dermatomyositis.

\section{Case Reports}

Case 1. A 51-year-old man had a 1-year history of dermatomyositis with proximal myopathy, heliotrope rash, Gottron's sign and positive anti-Jo-1 antibody. He was successfully treated with systemic corticosteroids. However, during the decreased doses of oral prednisolone to $5 \mathrm{mg} /$ day, itchy eruptions appeared on his lower back and upper extremities. Each eruption was a bean-sized solitary papule (fig. 1a). Many of the papules had erosions or crusts due to excoriation. Some papules were arranged linearly or clustered to form small plaques. Steroid ointment was ineffective. He also had other cutaneous manifestations of dermatomyositis such as heliotrope rash, flagellate erythema of the upper back, periungual erythema and mechanic's hands. Histologically, in the biopsy specimen of a papule of the lower trunk, there was a dermal cellular infiltrate comprising lymphocytes and histiocytes around superficial vessels with a weak irregular acanthosis (fig. 1b). Faint deposition of mucin was detected beneath the epidermis as assessed by Alcian blue staining. Laboratory findings revealed elevated levels of serum creatine kinase, 1,022 U/ml (normal 35-175). Pruritic eruptions were dramatically improved after the increase in doses of oral prednisolone. An intense survey revealed no internal malignancy, while an interstitial pneumonia which seemed to be caused by dermatomyositis was detected.

Case 2. An 80-year-old man had been diagnosed as having dermatomyositis with proximal myopathy, poikiloderma and a high value of creatine kinase $(5,191 \mathrm{U} / \mathrm{ml}) 1$ year before the consultation at our clinic. Systemic corticosteroid (prednisolone 60 $\mathrm{mg} /$ day) was successful to relieve these symptoms. After oral prednisolone had been reduced to a dose as low as $4 \mathrm{mg} /$ day, pruritic eruptions appeared on his back. Eruptions were bean-sized to fingertip-sized elevated urticarial papules, which were distributed solitarily around his waist. Some of the papules were clustered to form elevated plaques (fig. 2). Eruptions were successfully treated with topical corticosteroid. No malignancy was found during our observation.
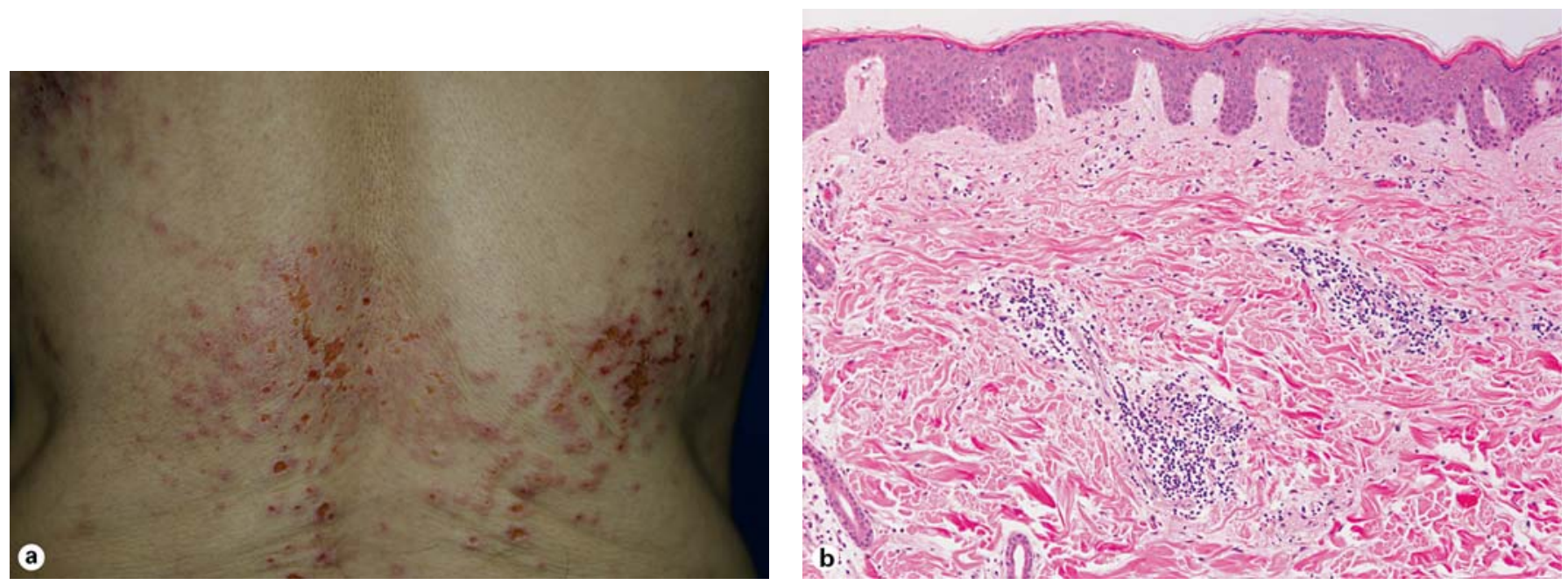

Fig. 1. Prurigo eruptions in case 1. a Itchy papules were distributed on the lower back. Some of the papules tended to aggregate to form a plaque. $\mathbf{b}$ Histological feature of papule. HE. $\times 40$. 


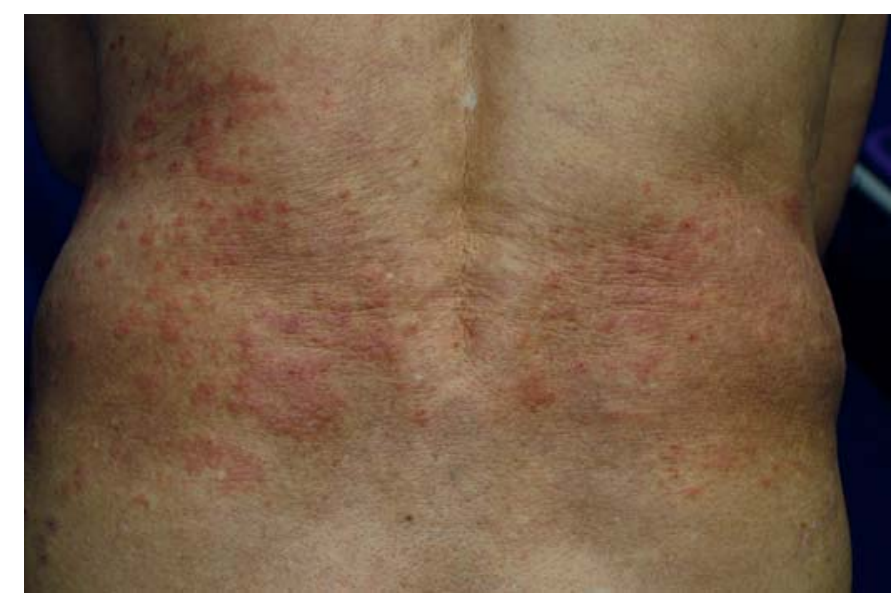

Fig. 2. Clinical manifestations of case 2 .

\section{Discussion}

We diagnosed the eruption of our cases as prurigo based on the following reasons. First, each urticarial papule was generally distributed solitarily. Second, each papule caused intense pruritus with excoriation. In addition, histologically, there was a lymphocytic infiltration mixed with a few eosinophils around blood vessels in the upper dermis.

Prurigo is a condition of nodular cutaneous lesions that are characterized by an intense itch. It is generally classified into three forms: acute, subacute and chronic [9]. While most of the subacute and chronic forms appear to be idiopathic, internal malignancy has been suggested to be one of the etiological precipitating factors.

In our cases, no malignant tumors could be detected. Notably, prurigo eruptions seemed to be well correlated with the disease activity of dermatomyositis. Thus, it was postulated that prurigo reactions occurred in association with dermatomyositis. Prurigolike lesions have also been reported to occur in patients with disseminated discoid lupus erythematosus, systemic scleroderma and adult-onset Still disease [10].
The differential diagnosis involved flagellate erythema in dermatomyositis. Flagellate erythema is distinct from a prurigo reaction in that it is characterized by linear erythema caused by scratching and is usually found on the shoulders or upper trunk [11]. Interestingly, however, some of the prurigo papules in our cases were arranged linearly (fig. 1a, 2), resembling a linear pattern of flagellate erythema. Therefore, these two types of eruptions may share similarities in their pathological mechanisms.

\section{References}

1 Caro I: Dermatomyositis. Semin Cutan Med Surg 2001;20:38-45.

2 Callen JP, Wortmann RL: Dermatomyositis. Clin Dermatol 2006;24: 363-373.

-3 McCollough ML, Cockerell CJ: Vesiculo-bullous dermatomyositis. Am J Dermatopathol 1998;20:170-174.

4 Ghali FE, Groben PA, McCauliffe DP: Panniculitis in juvenile dermatomyositis. Pediatr Dermatol 1999;16:270-272.

5 Kaufmann R, Greiner D, Schmidt P, Wolter M: Dermatomyositis presenting as plaque-like mucinosis. Br J Dermatol 1998;138:889-892.

-6 Requena L, Grilli L, Soriano L, Escalonilla P, Farina C, Martin L: Dermatomyositis with pityriasis rubra pilaris-like eruption: a little-known distinctive cutaneous manifestation of dermatomyositis. Br J Dermatol 1997;136:768-771.

7 Polat M, Lenk N, Üstün H, Öztaş P, Artüz F, Alli N: Dermatomyositis with a pityriasis rubra pilaris-like eruption: an uncommon cutaneous manifestation in dermatomyositis. Pediatr Dermatol 2007;2:151-154.

8 Okiyama N, Kohsaka H, Ueda N, Satoh T, Katayama I, Nishioka K, Yokozeki H: Seborrheic area erythema as a common skin manifestation in Japanese patients with dermatomyositis. Dermatology 2008;217:374377.

9 Wallengren J: Prurigo: diagnosis and management. Am J Clin Dermatol 2004;5:85-95.

10 Kaur S, Bambery P, Dhar S: Persistent dermal plaque lesions in adult onset Still's disease. Dermatology 1994;188:241-242.

11 Nousari HC, Ha VT, Leman SD, Provost TT, Tausk FA: 'Centripetal flagellate erythema': a cutaneous manifestation associated with dermatomyositis. J Rheumatol 1999;26:610-615.

Dr. Tetsuya Higuchi

Department of Dermatology, Graduate School

Tokyo Medical and Dental University

1-5-45 Yushima, Bunkyo-ku

Tokyo 113-8519 (Japan)

Tel. +81 35803 5284, Fax +81358035289

E-Mail tetsuyah.derm@tmd.ac.jp 